\title{
Ecological disturbance in tropical peatlands prior to marine
}

\section{Permian-Triassic mass extinction}

\author{
Daoliang Chu' ${ }^{1 *}$, Stephen E. Grasby ${ }^{2}$, Haijun Song1, Jacopo Dal Corso ${ }^{3}$, Yao Wang ${ }^{1}$, Tamsin A. \\ Mather $^{4}$, Yuyang Wu ${ }^{1}$, Huyue Song ${ }^{1}$, Wenchao Shu ${ }^{1}$, Jinnan Tong ${ }^{1}$, and Paul B. Wignall ${ }^{3 *}$ \\ ${ }^{1}$ State Key Laboratory of Biogeology and Environmental Geology, School of Earth Sciences, China \\ University of Geosciences, Wuhan 430074, China \\ ${ }^{2}$ Geological Survey of Canada, Natural Resources Canada, Calgary, Alberta T2L2A7, Canada \\ ${ }^{3}$ School of Earth and Environment, University of Leeds, Leeds LS2 9JT, UK \\ ${ }^{4}$ Department of Earth Sciences, University of Oxford, South Parks Road, Oxford, UK \\ *E-mail: chudl@cug.edu.cn; p.b.wignall@leeds.ac.uk
}

\section{Sample processing}

To avoid the possibility of variable transport capacity associated with the different processes that led to the facies variations, only samples from mudstones were processed. Forty-two unweathered samples in total were processed using acid maceration techniques. Firstly, we broke the original bulk samples ( $\sim 40 \mathrm{~g}$ of each sample, Table DR2) into $1.5-2 \mathrm{~cm}$ pieces before acid treatment with the smaller pieces or powdery removed. Then, treatment with cold hydrochloric acid $(36 \% \mathrm{HCl})$ removed carbonate, and samples were then rinsed with ultrapure water. Subsequently, treatment with cold concentrated hydrofluoric acid (39\% HF) for $24 \mathrm{~h}$ removed silicates, and then were rinsed with ultrapure water again. Acid treatments were repeated twice. The process was completed with a final treatment in cold $\mathrm{HCl}$ for $24 \mathrm{~h}$ to avoid calcium fluoride precipitation. Finally, samples were rinsed with ultrapure water until a neutral $\mathrm{pH}$ was achieved. The residue was sieved over a $100-\mu \mathrm{m}-\mathrm{mesh}$ screen and both size fractions were collected. The $>100 \mu \mathrm{m}$ size fractions of all samples were analyzed using a binocular microscope and the numbers of charcoal particles observed were quantified to represent charcoal abundance. Selected samples of charcoal were examined using a scanning electron microscope (SEM) to confirm their identification as charcoal. The $<100 \mu \mathrm{m}$ size fractions was quantified by evenly dispersing the organic particles in a known quantity of water. A known volume was then pipetted and made into slides using standard palynological techniques. The palynofacies analysis of each sample was conducted, quantifying the abundance of pollen and spores, plant cuticle, amorphous organic matter and coalified particles.

For bulk organic carbon isotope analyses, 35 samples were selected from the ZK4703 core section. Each sample was crushed to fine powder ( $<200 \mathrm{mesh}$ ), and $\sim 2 \mathrm{~g}$ of powder was weighed and placed 
into a centrifuge tube. The powdered samples were reacted with $4 \mathrm{~mol} / \mathrm{L} \mathrm{HCl}$ for $24 \mathrm{~h}$ to remove carbonate, then rinsed with ultrapure water repeatedly until neutralized, and finally dried at $35^{\circ} \mathrm{C}$. For cuticles and charcoal organic carbon isotopes analyses, 23 and 19 samples were selected, respectively. The $\delta^{13} \mathrm{C}_{\text {org }}$ values of the treated samples were obtained using an elemental analyzer (EA) coupled with an isotope ratio mass spectrometer (Thermo Finnegan DeltaV) at the China University of Geosciences (Wuhan). The results were calibrated using USGS standards: USGS40 $\left(\delta^{13} \mathrm{C}=-26.39 \%\right.$ ) and UREA $\left(\delta^{13} \mathrm{C}=-37.32 \%\right.$ ). The analytical precision of $\delta^{13} \mathrm{C}_{\text {org }}$ was better than $\pm 0.2 \%$. Organic carbon isotope values are given in per mille relative to VPDB. For total organic carbon (TOC) analyses, about $\sim 4 \mathrm{~g}$ of powdered sample was put into the $30 \mathrm{~mL}$ tube, then $50 \% \mathrm{HCl}$ was injected to dissolve carbonate minerals. After multiple centrifugal and lyophilization, the residue was analyzed for total organic carbon (TOC). A Multi EA 4000-analyzer was used for TOC and total sulfur concentrations (TS) determination at the China University of Geosciences (Wuhan), yielding an analytical accuracy of $1.5 \%$ and $2 \%$ of the reported values, respectively. Major element abundances of ZK4703 samples were determined by an Inductively Coupled Plasma source mass spectrometer with Agilent 7700e at Wuhan SampleSolution Analytical Technology Co. Ltd. For the samples from ZK4703, Hg content was measured using a LECO AMA254 mercury analyzer at the China University of Geosciences (Wuhan). Data reliability was assured by use of international standard 502-685 with $\mathrm{Hg}$ concentration of $40 \pm 8$ ppb, and analytic precision was within 5\%. For the samples from Chinahe, $\mathrm{Hg}$ content was measured using a Lumex RA-915 Portable Mercury Analyzer with PYRO-915 Pyrolyzer at the University of Oxford. The machine was calibrated before use with standards of NIST2587 with Hg concentration of $290 \pm 9$ ppb, analytic precision was within $6 \%$.

\section{Studied sections}

Chinahe section $\left(26.13077^{\circ} \mathrm{N}, 104.35637^{\circ} \mathrm{E}\right)$ is located in Chinahe Village of the Tianba town, 30 $\mathrm{km}$ southeastern of Xuanwei City. It comprises a continuously exposed outcrop of the upper part of the Xuanwei Formation, the Kayitou Formation and the lower part of the Dongchuan Formation. The successions within the Xuanwei Formation were deposited chiefly in an alluvial-plain to fluvial and lacustrine environments. The Gigantopteris flora commonly ranges to immediately above the last occurrence of coal in the Xuanwei Formation. The Kayitou Formation is similar to the underlying Xuanwei Formation, mainly comprising grey-green mudstone and sandstone. The difference between the Kayitou and Xuanwei formations is that the former contains no coal beds. The rocks of the Kayitou Formation, in ascending order, are green sandstone, siltstone, and mudstone in the lower part, brownish-yellow-green siltstone, and mudstone in the middle part, and purple-red siltstone increasing 
in proportion upwards in the upper part. The Dongchuan Formation is characterized by a thick succession of purple-red siliciclastic sandstone, siltstone and mudstone.

The ZK4703 core $\left(25.54151^{\circ} \mathrm{N}, 104.28994^{\circ} \mathrm{E}\right)$, was drilled in Anzichong Viliage of Dahe Town, $15 \mathrm{~km}$ south of Fuyuan County, Qujing City. It comprises a continuously successions of the Xuanwei Formation, the Kayitou Formation and the Dongchuan Formation. The Xuanwei Formation lies unconformably on the Emeishan basalts and consists mainly of siliciclastic sandstone beds with intercalations of organic-rich mudstone and coal beds. Large pieces of Gigantopteris leaves are commonly observed in the core. The Kayitou Formation is similar to the underlying Xuanwei Formation, but shows no coal beds. The Dongchuan Formation is characterized by a thick succession (over $500 \mathrm{~m}$ thick) of purple-red siliciclastic sandstones, siltstones and mudstones.

\section{$\mathrm{Hg} / \mathrm{TOC}, \mathrm{Hg} / \mathrm{Al}$ and $\mathrm{Hg} / \mathrm{TS}$}

$\mathrm{Hg}$ can be associated with the TOC, sulfide or clay fractions. In the present study, we measured the TOC, Al and S contents of ZK4703 section to assess the effect of organic matter burial, redox

condition and clay abundance on the Hg enrichment. ZK4703 core has low total sulfur contents, mostly $<0.15 \%$ which is too low to evaluate. They show no covariation with $\mathrm{Hg}$ concentrations. The Al contents varies little and the correlation with $\mathrm{Hg}$ concentrations is poor $\left(\mathrm{R}^{2}=0.1358, \mathrm{P}=0.03\right)$, indicating that $\mathrm{Hg}$ fluctuations are not controlled primarily by clay content. The TOC concentrations average $\sim 1.3 \%$ in the Xuanwei Formation and $\sim 0.4 \%$ in the Kayitou Formation. After normalizing $\mathrm{Hg}$ concentrations by TOC values, there is no significant correlation (Chinahe, $\mathrm{R}^{2}=0.0032, \mathrm{P}=0.68$; $\mathrm{ZK} 4703, \mathrm{R}^{2}=0.0384, \mathrm{P}=0.25$ ), suggesting that $\mathrm{Hg}$ peaks are not linked to organic matter scavenging. The strong noncorrelative trends of $\mathrm{Hg}, \mathrm{TOC}$, and $\mathrm{Al}$ through time suggest that the $\mathrm{Hg}$ anomalies are not linked to increased organic matter burial and/or clay abundance. 

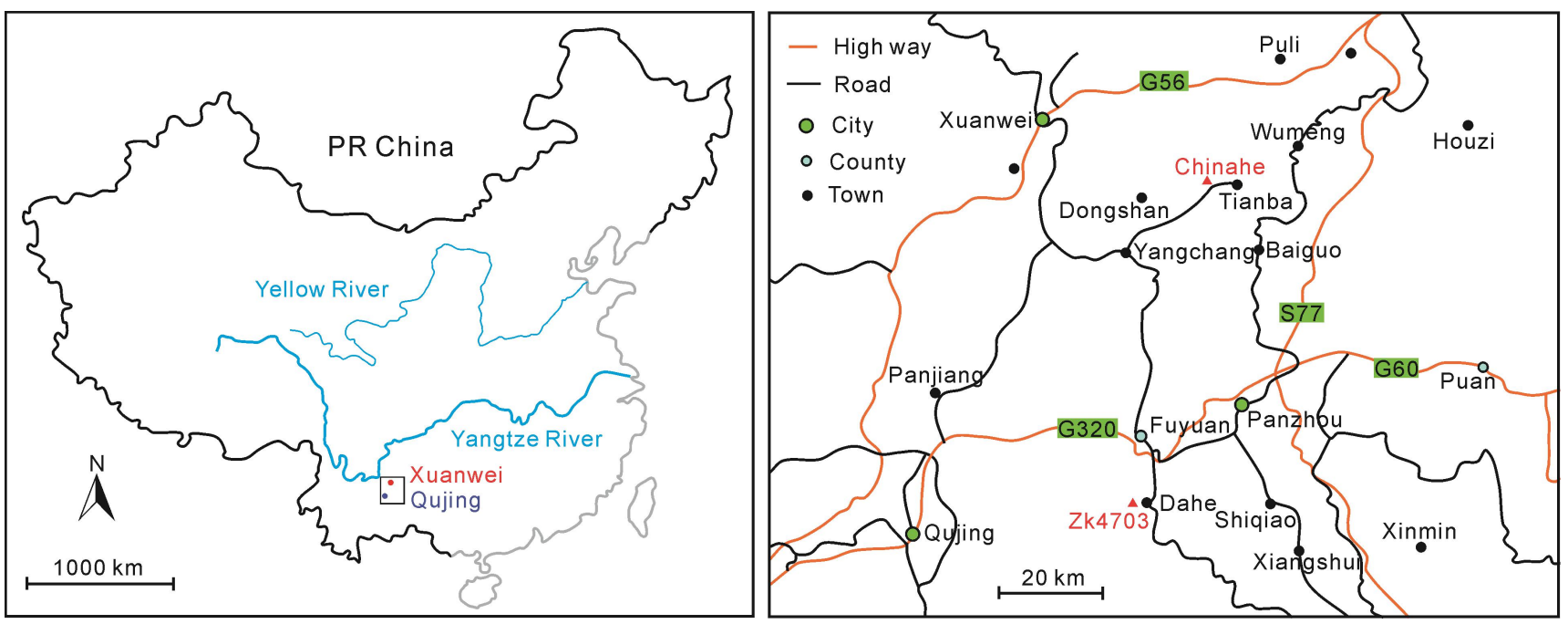

Figure DR1. Location map of the studied section. Chinahe section $\left(26.13077^{\circ} \mathrm{N}, 104.35637^{\circ} \mathrm{E}\right)$ is located in the Chinahe Viliage of the Tianba town, Xuanwei City. ZK4703 core $\left(25.54151^{\circ} \mathrm{N}\right.$, 104.28994 ${ }^{\circ}$ ), drilled in Anzichong Viliage of Dahe Town, Qujing City. 

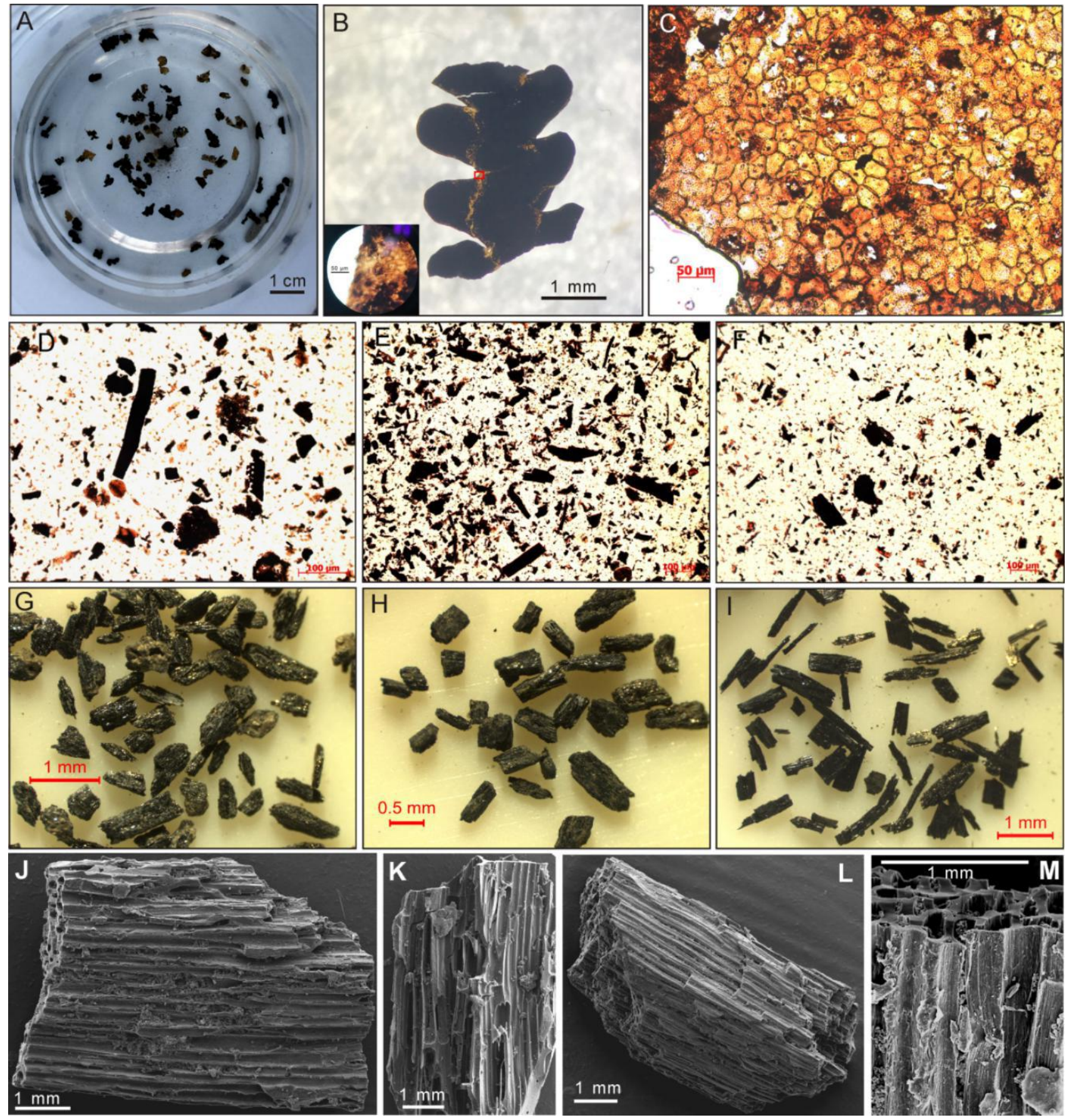

Figure DR2. Cuticle and charcoal particles under binocular microscope and SEM. 

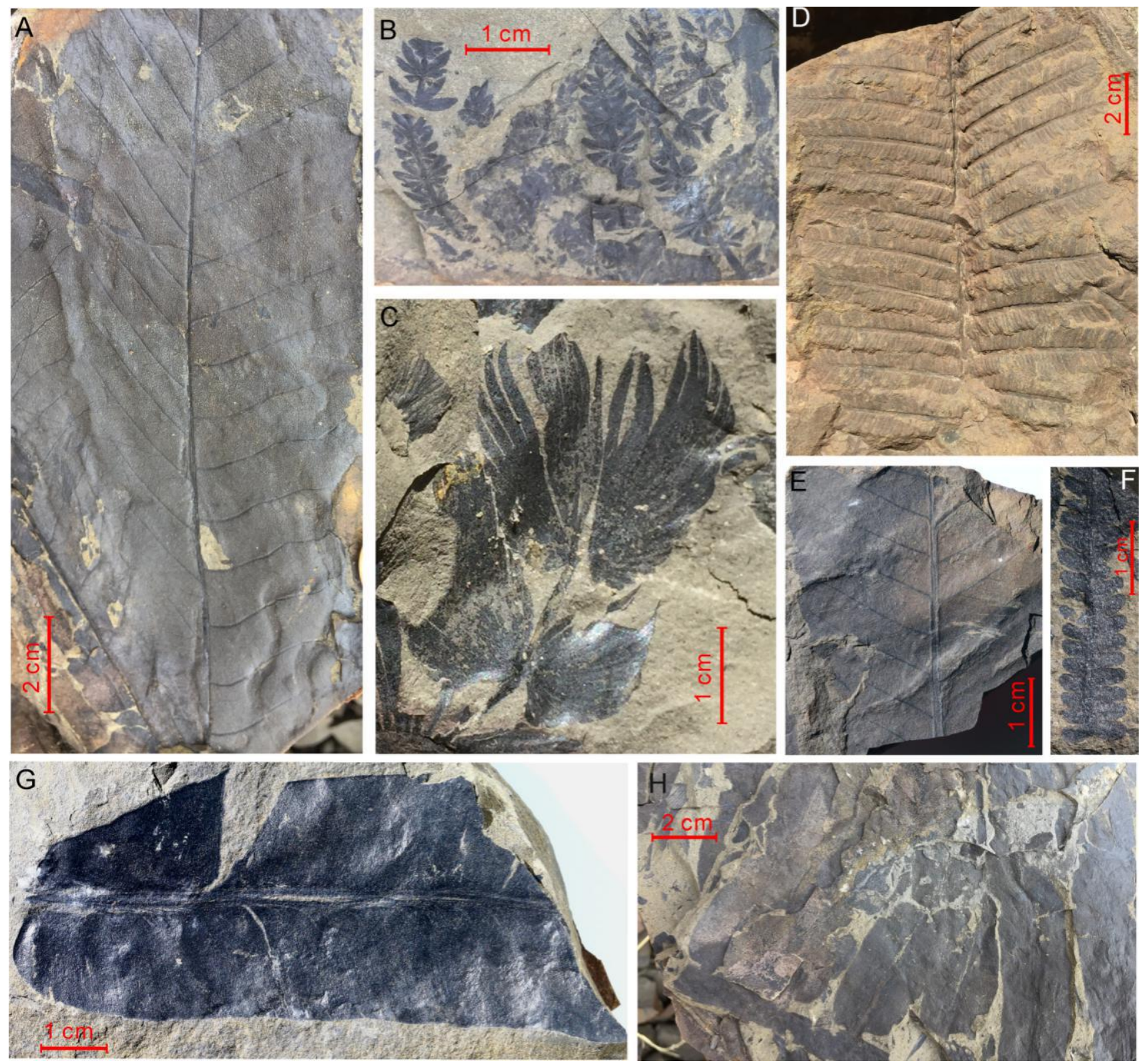

Figure DR3. Typical Gigantopteris flora from the Xuanwei Formation of the Chinahe section. A: Gigantopteris dictyophylloides; B: Annularia pingloensis; C: Lobatannularia sp.; D: Pecopteris marginata; E: Gigantonoclea guizhouensis; F: Pecopteris sp.; G: Compsopteris contracta; H: Abundant plant leaf fossils preserved on the same bedding surface 

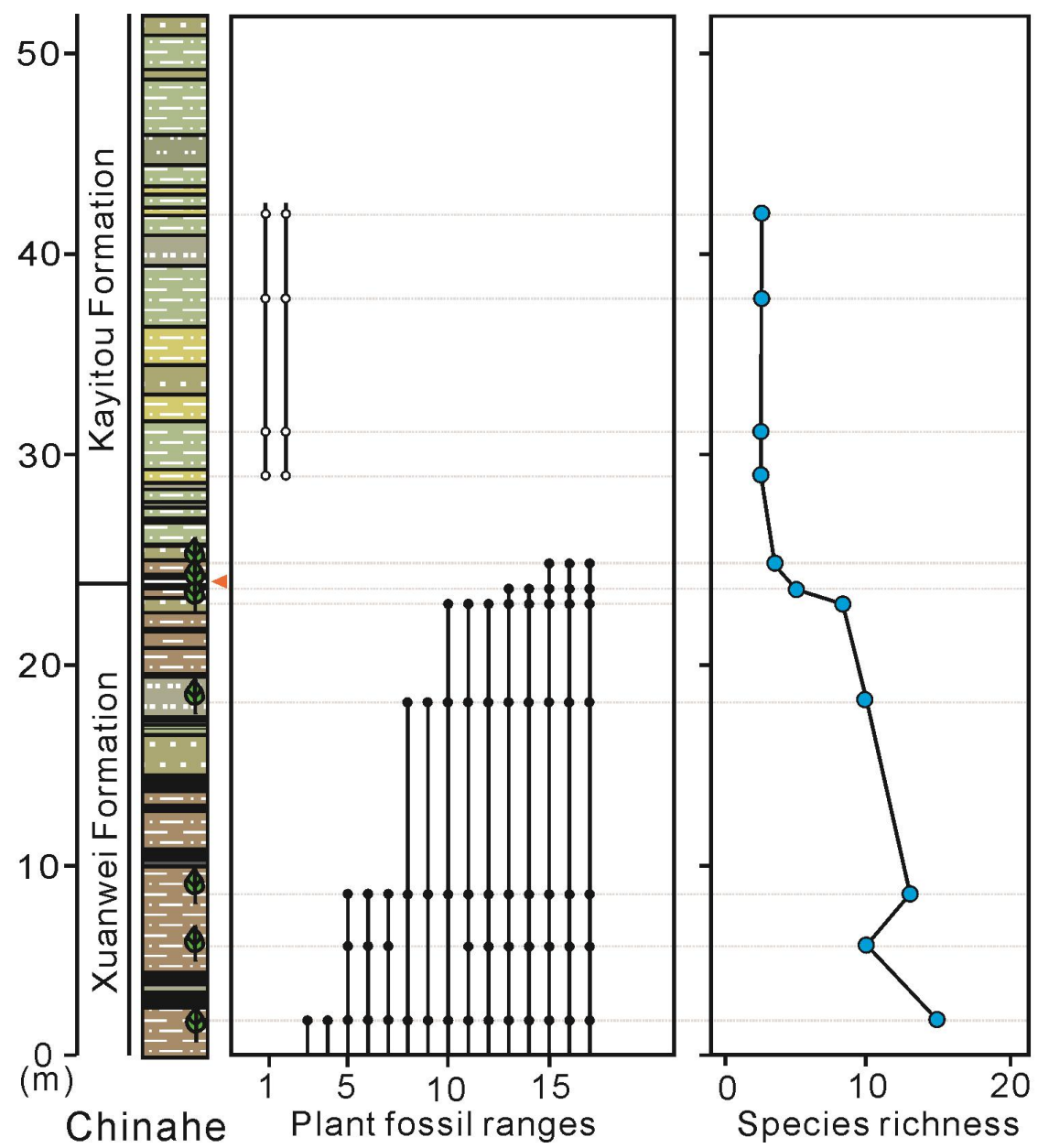

Gigantopteris flora

$\cdots$

Sandstone

…

Siltstone

E-G

Silty mudstone

E-

Mudstone

ב

Coal bed

Ash bed

Figure DR4. The plant fossil ranges and species richness of the Chinahe section. 1. Peltaspermum sp.;

2. Annalepis sp.; 3. Compsopteris contracta; 4. Fascipteris densata; 5. Cladophlebis permica; 6. Annularia pingloensis; 7. Compsopteris sp.; 8. Lobatannularia heianensis; 9. Pecopteris marginata; 10. Lobatannularia cathaysiana; 11. Pecopteris guizhouensis; 12. Rajahia guizhouensis; 13. Gigantonoclea sp.; 14. Stigmaria sp.; 15. Gigantonoclea guizhouensis; 16. Gigantopteris dictyophylloides; 17. Pecopteris sp.. 

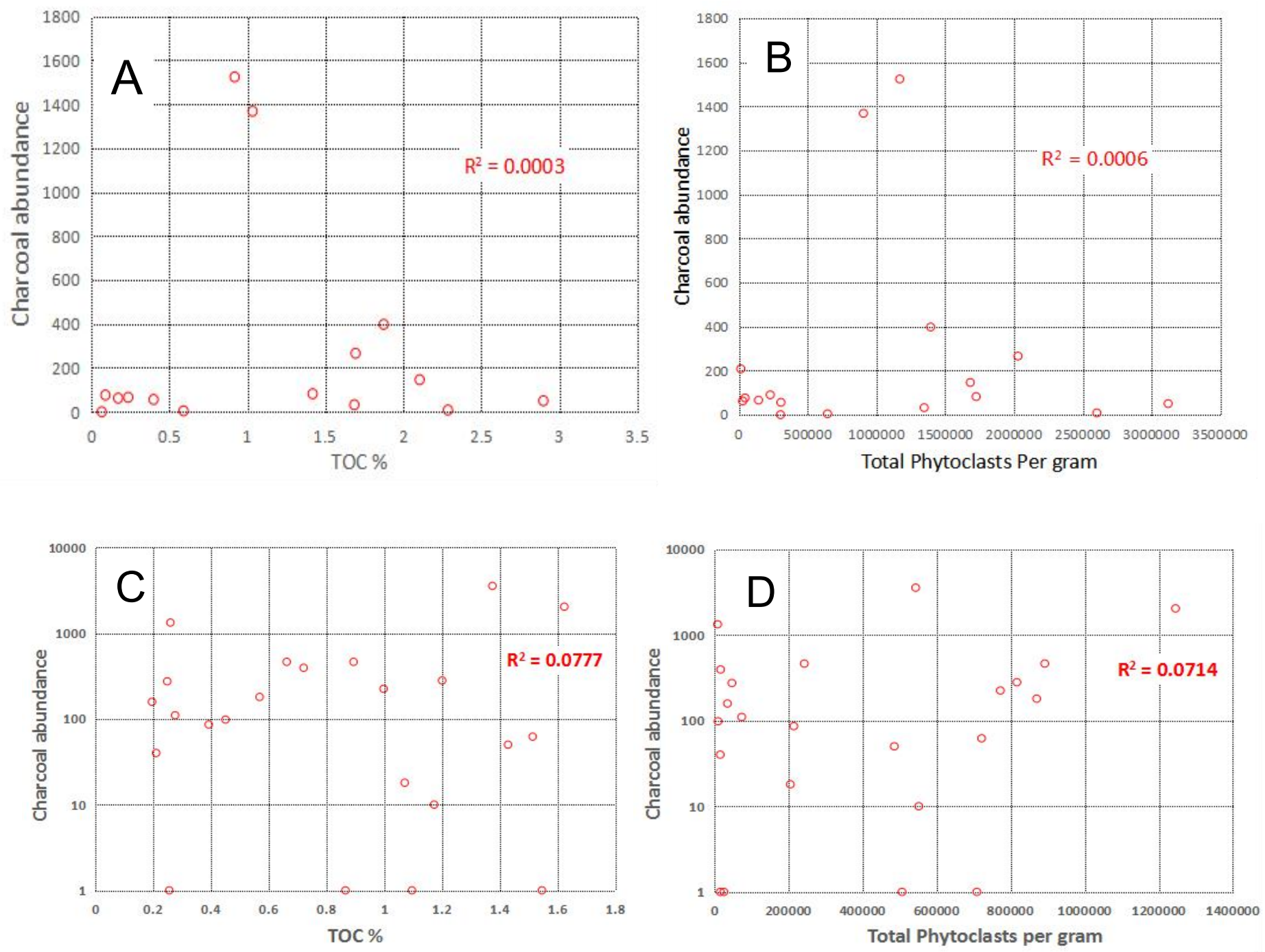

Figure DR5. Charcoal abundance (charcoal particles per $100 \mathrm{~g}$ ) versus TOC and charcoal abundance versus phytoclasts abundance, showing variations in preserved phytoclasts and TOC show little correlation with the variations in charcoal abundance. A, B: Chinahe; C, D: ZK4703. 

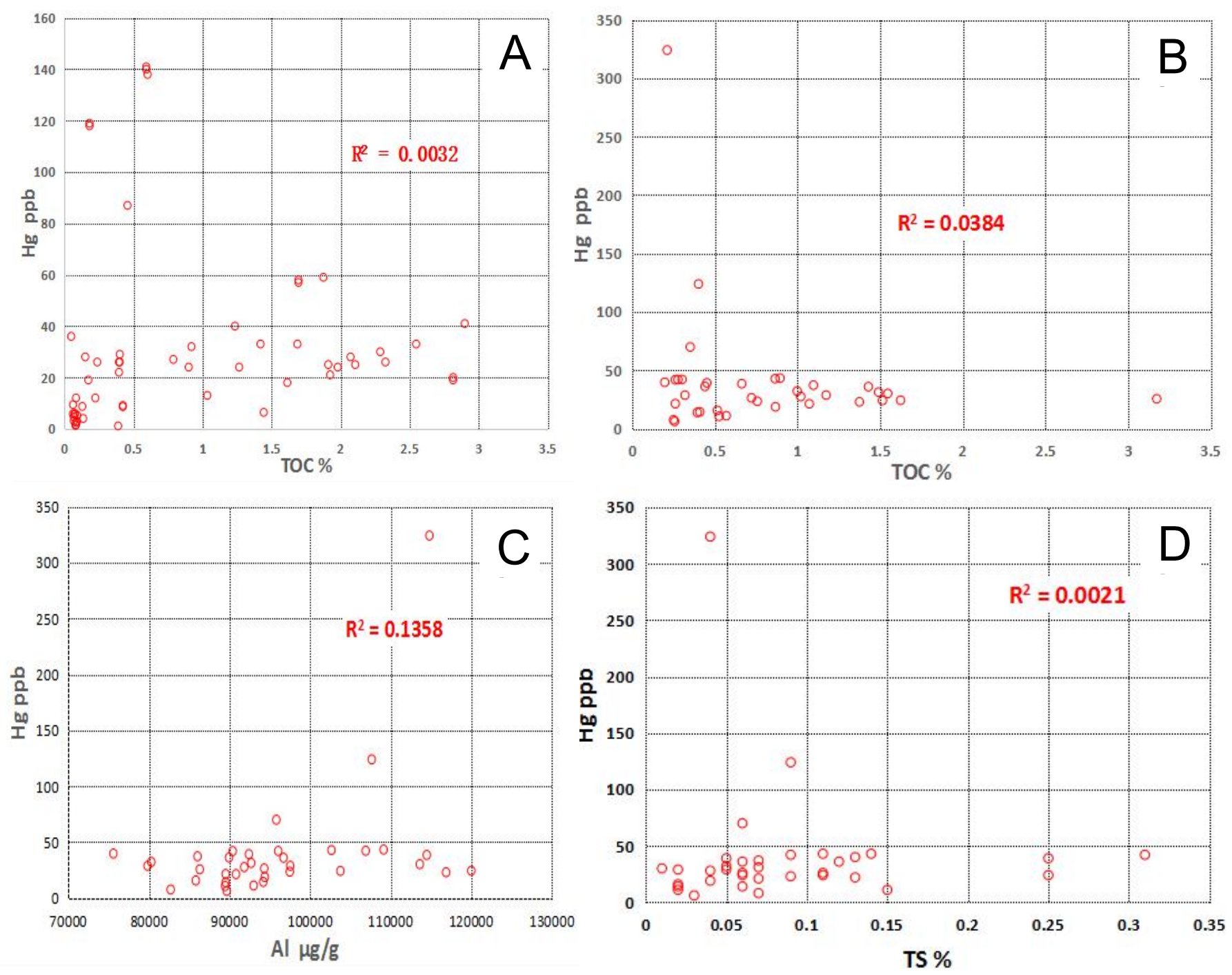

Figure DR6. A: Hg content versus TOC at Chinahe, showing weak correlation; B: Hg content versus TOC at ZK4703, showing weak correlation; C: Hg content versus Al content at ZK4703, showing weak correlation; D: Hg content versus TS at ZK4703, showing weak correlation. 

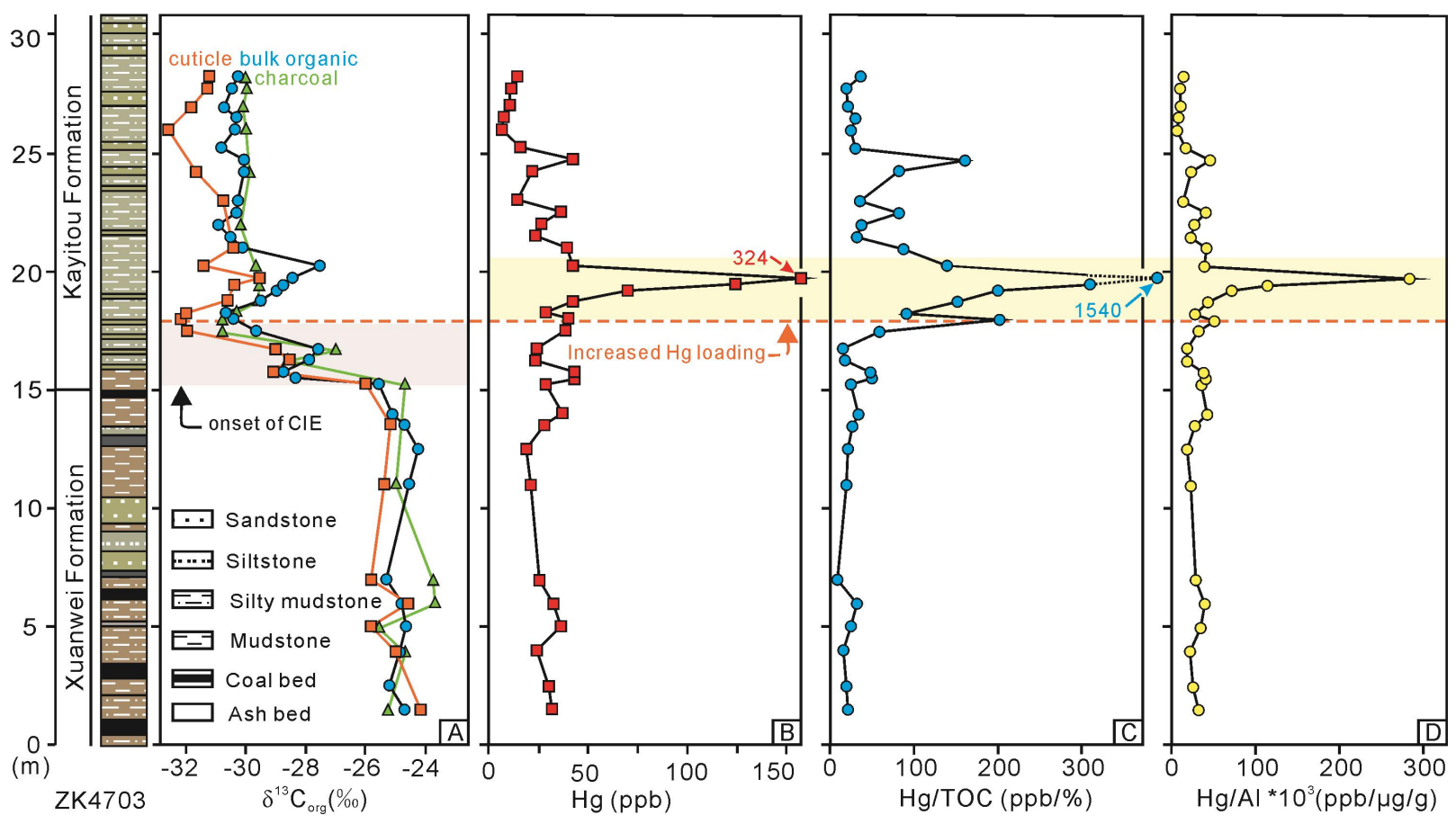

Figure DR7. Organic carbon isotopes, $\mathrm{Hg}$ concentration, $\mathrm{Hg} / \mathrm{TOC}$ and $\mathrm{Hg} / \mathrm{Al}$ ratios in the $\mathrm{ZK} 4703$ core sections. 
Table DR1. Hg, TOC, TS, Al, organic carbon isotopes and charcoal abundance (particles per $100 \mathrm{~g}$ bulk rock) data from ZK4703.

\begin{tabular}{|c|c|c|c|c|c|c|c|c|c|c|c|}
\hline \multirow{2}{*}{ Sample } & \multirow{2}{*}{$\begin{array}{c}\text { Height } \\
\text { (m) }\end{array}$} & \multirow{2}{*}{$\begin{array}{l}\mathrm{Hg} \\
(\mathrm{ppb})\end{array}$} & \multirow{2}{*}{$\begin{array}{c}\text { TOC } \\
\%\end{array}$} & \multirow{2}{*}{$\begin{array}{l}\mathrm{TS} \\
\%\end{array}$} & \multirow{2}{*}{$\begin{array}{c}\mathrm{Al} \\
\mu \mathrm{g} / \mathrm{g}\end{array}$} & \multirow{2}{*}{$\begin{array}{l}\mathrm{Hg} / \mathrm{TOC} \\
(\mathrm{ppb} / \%)\end{array}$} & \multirow{2}{*}{$\begin{array}{c}\mathrm{Hg} / \mathrm{Al}^{*} 10^{3} \\
(\mathrm{ppb} / \\
\mu \mathrm{g} / \mathrm{g})\end{array}$} & \multicolumn{3}{|c|}{ Organic carbon isotope $(\%)$} & \multirow{2}{*}{$\begin{array}{l}\text { Charcoal } \\
\text { abundance }\end{array}$} \\
\hline & & & & & & & & Bulk OM & Cuticles & Charcoal & \\
\hline $\mathrm{ZK}-3$ & 1.50 & 31 & 1.49 & 0.07 & 928 & 21 & 34 & -24.67 & -24.20 & -25.15 & 440 \\
\hline ZK-5 & 2.50 & 30 & 1.55 & 0.01 & 1136 & 20 & 27 & -25.18 & $\mathrm{~N}$ & $\mathrm{~N}$ & 0 \\
\hline ZK-8 & 4.00 & 24 & 1.51 & 0.06 & 1038 & 16 & 23 & -24.81 & -24.93 & -24.68 & 62 \\
\hline ZK-10 & 5.00 & 36 & 1.43 & 0.12 & 967 & 25 & 37 & -24.65 & -25.79 & -25.57 & 50 \\
\hline $\mathrm{ZK}-12$ & 6.00 & 32 & 1.00 & 0.05 & 803 & 32 & 40 & -24.81 & -24.53 & -23.70 & 224 \\
\hline ZK-14 & 7.00 & 26 & 3.17 & 0.06 & 863 & 8 & 29 & -25.30 & -25.81 & -23.73 & 280 \\
\hline ZK-16 & 9.00 & $\mathrm{~N}$ & $\mathrm{~N}$ & $\mathrm{~N}$ & $\mathrm{~N}$ & $\mathrm{~N}$ & $\mathrm{~N}$ & $\mathrm{~N}$ & $\mathrm{~N}$ & $\mathrm{~N}$ & 28 \\
\hline ZK-17 & 11.00 & 21 & 1.07 & 0.07 & 908 & 20 & 24 & -24.52 & -25.35 & -25.09 & 18 \\
\hline ZK-19 & 12.00 & $\mathrm{~N}$ & $\mathrm{~N}$ & $\mathrm{~N}$ & $\mathrm{~N}$ & $\mathrm{~N}$ & $\mathrm{~N}$ & $\mathrm{~N}$ & $\mathrm{~N}$ & $\mathrm{~N}$ & 0 \\
\hline $\mathrm{ZK}-20$ & 12.50 & 19 & 0.87 & 0.04 & 944 & 22 & 20 & -24.23 & $\mathrm{~N}$ & $\mathrm{~N}$ & $\mathrm{~N}$ \\
\hline ZK-21 & 13.00 & $\mathrm{~N}$ & $\mathrm{~N}$ & $\mathrm{~N}$ & $\mathrm{~N}$ & $\mathrm{~N}$ & $\mathrm{~N}$ & $\mathrm{~N}$ & $\mathrm{~N}$ & $\mathrm{~N}$ & 8 \\
\hline $\mathrm{ZK}-22$ & 13.50 & 28 & 1.02 & 0.04 & 918 & 27 & 30 & -24.68 & $\mathrm{~N}$ & $\mathrm{~N}$ & $\mathrm{~N}$ \\
\hline ZK-23 & 14.00 & 37 & 1.10 & 0.07 & 860 & 34 & 43 & -25.07 & $\mathrm{~N}$ & $\mathrm{~N}$ & 0 \\
\hline ZK-25 & 15.00 & $\mathrm{~N}$ & $\mathrm{~N}$ & $\mathrm{~N}$ & $\mathrm{~N}$ & $\mathrm{~N}$ & $\mathrm{~N}$ & $\mathrm{~N}$ & $\mathrm{~N}$ & $\mathrm{~N}$ & 708 \\
\hline ZK-26 & 15.25 & 29 & 1.17 & 0.05 & 798 & 25 & 36 & -25.55 & -25.99 & -24.70 & 10 \\
\hline $\mathrm{ZK}-27$ & 15.50 & 43 & 0.86 & 0.14 & 1027 & 50 & 41 & -28.33 & $\mathrm{~N}$ & $\mathrm{~N}$ & $\mathrm{~N}$ \\
\hline $\mathrm{ZK}-28$ & 15.75 & 43 & 0.89 & 0.11 & 1091 & 48 & 39 & -28.72 & -29.13 & $\mathrm{~N}$ & 462 \\
\hline ZK-29 & 16.00 & $\mathrm{~N}$ & $\mathrm{~N}$ & $\mathrm{~N}$ & $\mathrm{~N}$ & $\mathrm{~N}$ & $\mathrm{~N}$ & $\mathrm{~N}$ & $\mathrm{~N}$ & $\mathrm{~N}$ & 1394 \\
\hline ZK-30 & 16.25 & 23 & 1.37 & 0.09 & 1169 & 17 & 20 & -27.87 & -28.58 & -28.65 & $\mathrm{~N}$ \\
\hline $\mathrm{ZK}-31$ & 16.50 & $\mathrm{~N}$ & $\mathrm{~N}$ & $\mathrm{~N}$ & $\mathrm{~N}$ & $\mathrm{~N}$ & $\mathrm{~N}$ & $\mathrm{~N}$ & $\mathrm{~N}$ & $\mathrm{~N}$ & 2482 \\
\hline ZK-32 & 16.75 & 24 & 1.62 & 0.25 & 1200 & 15 & 20 & -27.57 & -29.08 & -27.01 & 2034 \\
\hline ZK-33 & 17.00 & $\mathrm{~N}$ & $\mathrm{~N}$ & $\mathrm{~N}$ & $\mathrm{~N}$ & $\mathrm{~N}$ & $\mathrm{~N}$ & $\mathrm{~N}$ & $\mathrm{~N}$ & $\mathrm{~N}$ & 38 \\
\hline ZK-34 & 17.25 & $\mathrm{~N}$ & $\mathrm{~N}$ & $\mathrm{~N}$ & $\mathrm{~N}$ & $\mathrm{~N}$ & $\mathrm{~N}$ & $\mathrm{~N}$ & $\mathrm{~N}$ & $\mathrm{~N}$ & 462 \\
\hline ZK-35 & 17.50 & 39 & 0.66 & 0.25 & 1145 & 58 & 34 & -29.64 & -31.94 & -30.79 & 372 \\
\hline ZK-37 & 18.00 & 40 & 0.20 & 0.13 & 756 & 203 & 53 & -30.40 & -32.18 & -30.77 & 158 \\
\hline ZK-39 & 18.25 & 29 & 0.32 & 0.02 & 975 & 90 & 29 & -30.67 & -32.01 & -30.30 & 216 \\
\hline
\end{tabular}




\begin{tabular}{|c|c|c|c|c|c|c|c|c|c|c|c|}
\hline ZK-41 & 18.75 & 42 & 0.28 & 0.31 & 960 & 152 & 44 & -29.49 & -30.53 & -29.58 & 110 \\
\hline ZK-42 & 19.25 & 70 & 0.35 & 0.06 & 958 & 200 & 73 & -28.90 & $\mathrm{~N}$ & $\mathrm{~N}$ & 24 \\
\hline ZK-43 & 19.50 & 124 & 0.40 & 0.09 & 1077 & 310 & 115 & -28.50 & -30.29 & -29.51 & $\mathrm{~N}$ \\
\hline $\mathrm{ZK}-45$ & 19.75 & 324 & 0.21 & 0.04 & 1148 & 1540 & 282 & -28.44 & -29.42 & $\mathrm{~N}$ & 40 \\
\hline $\mathrm{ZK}-47$ & 20.25 & 42 & 0.30 & $\mathrm{~N}$ & 1069 & 140 & 39 & -27.54 & -31.37 & -29.68 & 152 \\
\hline ZK-49 & 20.75 & $\mathrm{~N}$ & $\mathrm{~N}$ & $\mathrm{~N}$ & $\mathrm{~N}$ & $\mathrm{~N}$ & $\mathrm{~N}$ & $\mathrm{~N}$ & $\mathrm{~N}$ & $\mathrm{~N}$ & 98 \\
\hline ZK-50 & 21.00 & 39 & 0.45 & 0.05 & 924 & 87 & 42 & -30.10 & -30.33 & $\mathrm{~N}$ & $\mathrm{~N}$ \\
\hline ZK-51 & 21.25 & $\mathrm{~N}$ & $\mathrm{~N}$ & $\mathrm{~N}$ & $\mathrm{~N}$ & $\mathrm{~N}$ & $\mathrm{~N}$ & $\mathrm{~N}$ & $\mathrm{~N}$ & $\mathrm{~N}$ & 280 \\
\hline ZK-52 & 21.50 & 24 & 0.76 & 0.11 & 975 & 31 & 24 & -30.50 & $\mathrm{~N}$ & $\mathrm{~N}$ & $\mathrm{~N}$ \\
\hline ZK-53 & 21.75 & $\mathrm{~N}$ & $\mathrm{~N}$ & $\mathrm{~N}$ & $\mathrm{~N}$ & $\mathrm{~N}$ & $\mathrm{~N}$ & $\mathrm{~N}$ & $\mathrm{~N}$ & $\mathrm{~N}$ & 394 \\
\hline ZK-54 & 22.00 & 26 & 0.72 & 0.11 & 943 & 37 & 28 & -30.92 & & -30.20 & $\mathrm{~N}$ \\
\hline ZK-55 & 22.25 & $\mathrm{~N}$ & $\mathrm{~N}$ & $\mathrm{~N}$ & $\mathrm{~N}$ & $\mathrm{~N}$ & $\mathrm{~N}$ & $\mathrm{~N}$ & $\mathrm{~N}$ & $\mathrm{~N}$ & 40 \\
\hline ZK-56 & 22.50 & 36 & 0.44 & 0.06 & 899 & 82 & 40 & -30.31 & $\mathrm{~N}$ & $\mathrm{~N}$ & $\mathrm{~N}$ \\
\hline ZK-57 & 22.75 & $\mathrm{~N}$ & $\mathrm{~N}$ & $\mathrm{~N}$ & $\mathrm{~N}$ & $\mathrm{~N}$ & $\mathrm{~N}$ & $\mathrm{~N}$ & $\mathrm{~N}$ & $\mathrm{~N}$ & 34 \\
\hline ZK-58 & 23.00 & 14 & 0.41 & 0.06 & 942 & 35 & 15 & -30.27 & -30.69 & $\mathrm{~N}$ & $\mathrm{~N}$ \\
\hline ZK-63 & 24.25 & 22 & 0.26 & 0.13 & 895 & 83 & 25 & -30.05 & -31.64 & -29.85 & $\mathrm{~N}$ \\
\hline ZK-65 & 24.75 & 42 & 0.26 & 0.09 & 904 & 162 & 46 & -30.04 & $\mathrm{~N}$ & $\mathrm{~N}$ & $\mathrm{~N}$ \\
\hline ZK-67 & 25.25 & 16 & 0.51 & 0.02 & 858 & 30 & 18 & -30.82 & $\mathrm{~N}$ & $\mathrm{~N}$ & 310 \\
\hline $\mathrm{ZK}-70$ & 26.00 & 6 & 0.26 & 0.03 & 897 & 25 & 7 & -30.36 & -32.58 & -30.05 & 670 \\
\hline $\mathrm{ZK}-72$ & 26.50 & 8 & 0.25 & 0.07 & 827 & 31 & 9 & -30.30 & $\mathrm{~N}$ & $\mathrm{~N}$ & 274 \\
\hline $\mathrm{ZK}-74$ & 27.00 & 11 & 0.52 & 0.02 & 895 & 20 & 11 & -30.74 & -31.81 & -30.10 & 34 \\
\hline $\mathrm{ZK}-77$ & 27.75 & 11 & 0.57 & 0.15 & 930 & 20 & 12 & -30.46 & -31.37 & -29.71 & 180 \\
\hline ZK-79 & 28.25 & 14 & 0.39 & 0.02 & 895 & 36 & 15 & -30.27 & -31.34 & -29.99 & 86 \\
\hline
\end{tabular}


Table DR2. Hg, TOC, organic carbon isotope values and charcoal abundance (particles per $100 \mathrm{~g}$ bulk rock) data from Chinahe.

\begin{tabular}{|c|c|c|c|c|c|c|c|}
\hline \multirow{2}{*}{ Sample } & \multirow{2}{*}{$\begin{array}{c}\text { Height } \\
\text { (m) }\end{array}$} & \multirow{2}{*}{$\begin{array}{c}\mathrm{Hg} \\
(\mathrm{ppb})\end{array}$} & \multirow{2}{*}{$\begin{array}{l}\text { TOC } \\
(\%)\end{array}$} & \multirow{2}{*}{$\begin{array}{l}\mathrm{Hg} / \mathrm{TOC} \\
(\mathrm{ppb} / \%)\end{array}$} & \multicolumn{2}{|c|}{ Organic carbon isotope $(\%)$} & \multirow{2}{*}{$\begin{array}{l}\text { Charcoal } \\
\text { abundance }\end{array}$} \\
\hline & & & & & Bulk OM & Charcoal & \\
\hline $\mathrm{CNH}-02+0$ & 2.5 & 27 & 0.79 & 34 & -24.23 & $\mathrm{~N}$ & 4 \\
\hline $\mathrm{CNH}-03+0.1$ & 3.5 & $\mathrm{~N}$ & $\mathrm{~N}$ & $\mathrm{~N}$ & -24.23 & $\mathrm{~N}$ & $\mathrm{~N}$ \\
\hline $\mathrm{CNH}-03+1$ & 4.4 & 33 & 1.42 & 23 & -24.54 & -23.71 & 82 \\
\hline $\mathrm{CNH}-03+2$ & 5.4 & 24 & 1.26 & 19 & -24.48 & $\mathrm{~N}$ & $\mathrm{~N}$ \\
\hline $\mathrm{CNH}-03+3.8$ & 7.2 & 21 & 1.92 & 11 & -25.49 & -24.45 & 282 \\
\hline CNH-03-0.7 & 8.7 & 18 & 1.61 & 11 & -24.42 & $\mathrm{~N}$ & $\mathrm{~N}$ \\
\hline $\mathrm{CNH}-04+1.3$ & 10.7 & 41 & 2.90 & 14 & -24.30 & -23.59 & 50 \\
\hline CNH-04-0.6 & 11.9 & 25 & 1.91 & 13 & -23.94 & $\mathrm{~N}$ & $\mathrm{~N}$ \\
\hline CNH-05+0.2 & 12.3 & $\mathrm{~N}$ & $\mathrm{~N}$ & $\mathrm{~N}$ & -24.17 & $\mathrm{~N}$ & $\mathrm{~N}$ \\
\hline CNH-05+0.3 & 12.4 & 28 & 2.07 & 14 & -24.02 & $\mathrm{~N}$ & $\mathrm{~N}$ \\
\hline CNH-06+0.1 & 13.3 & $\mathrm{~N}$ & $\mathrm{~N}$ & $\mathrm{~N}$ & -23.66 & $\mathrm{~N}$ & $\mathrm{~N}$ \\
\hline CNH-06-1 & 15.3 & 33 & 1.69 & 20 & -24.90 & $\mathrm{~N}$ & 32 \\
\hline $\mathrm{CNH}-07+0.2$ & 16.5 & 24 & 0.90 & 27 & -24.55 & -25.07 & $\mathrm{~N}$ \\
\hline CNH-07+1.2 & 17.5 & 20 & 2.81 & 7 & -25.20 & $\mathrm{~N}$ & 90 \\
\hline CNH-08+0.1 & 18.7 & $\mathrm{~N}$ & $\mathrm{~N}$ & $\mathrm{~N}$ & -25.46 & $\mathrm{~N}$ & $\mathrm{~N}$ \\
\hline CNH-08-0.3 & 19.7 & 8.6 & 0.42 & 20 & -25.29 & $\mathrm{~N}$ & $\mathrm{~N}$ \\
\hline $\mathrm{CNH}-09+0.3$ & 20.3 & 1.1 & 0.39 & 3 & -25.55 & $\mathrm{~N}$ & 52 \\
\hline CNH-09+1.2 & 21.2 & 24 & 1.98 & 12 & -25.29 & $\mathrm{~N}$ & $\mathrm{~N}$ \\
\hline CNH-09-0.9 & 21.55 & $\mathrm{~N}$ & $\mathrm{~N}$ & $\mathrm{~N}$ & -25.24 & $\mathrm{~N}$ & $\mathrm{~N}$ \\
\hline CNH-10+0.1 & 22.55 & 6.4 & 1.44 & 4 & -25.33 & -23.76 & 380 \\
\hline CNH$-10+0.4$ & 22.85 & 13 & 1.03 & 13 & -25.23 & -24.10 & 1368 \\
\hline CNH-11+0.1 & 23.05 & $\mathrm{~N}$ & $\mathrm{~N}$ & $\mathrm{~N}$ & -25.12 & $\mathrm{~N}$ & $\mathrm{~N}$ \\
\hline CNH- $12+0.2$ & 23.55 & 26 & 2.32 & 11 & -26.81 & -24.33 & 90 \\
\hline $\mathrm{CNH}-12+0.5$ & 23.85 & 25 & 2.10 & 12 & -26.74 & -25.57 & 146 \\
\hline $\mathrm{CNH}-12+0.75$ & 24.10 & $\mathrm{~N}$ & $\mathrm{~N}$ & $\mathrm{~N}$ & $\mathrm{~N}$ & -26.37 & $\mathrm{~N}$ \\
\hline CNH-12+1 & 24.35 & 33 & 2.55 & 13 & -26.52 & -26.70 & 704 \\
\hline
\end{tabular}




\begin{tabular}{|c|c|c|c|c|c|c|c|}
\hline CNH-12+1.3 & 24.65 & 40 & 1.23 & 32 & -26.43 & -25.59 & 294 \\
\hline CNH-12+1.6 & 24.95 & 32 & 0.92 & 35 & -27.51 & -26.73 & 1524 \\
\hline $\mathrm{CNH}-13+0$ & 25.25 & 30 & 2.29 & 13 & $\mathrm{~N}$ & -26.72 & 8 \\
\hline $\mathrm{CNH}-13+0.7$ & 25.95 & 57 & 1.69 & 34 & -27.18 & -28.60 & 266 \\
\hline CNH-13+1.1 & 26.35 & 59 & 1.87 & 31 & -26.97 & -27.23 & 395 \\
\hline $\mathrm{CNH}-13+1.2$ & 26.45 & $\mathrm{~N}$ & $\mathrm{~N}$ & $\mathrm{~N}$ & -28.40 & $\mathrm{~N}$ & $\mathrm{~N}$ \\
\hline $\mathrm{CNH}-13+1.5$ & 26.75 & 141 & 0.59 & 238 & -30.14 & -29.71 & 4 \\
\hline $\mathrm{CNH}-13+2.3$ & 27.55 & 87 & 0.46 & 191 & -28.31 & $\mathrm{~N}$ & 6 \\
\hline CNH-14+0.1 & 28.85 & 29 & 0.40 & 73 & -28.61 & $\mathrm{~N}$ & 56 \\
\hline CNH-14+0.8 & 29.55 & 22 & 0.39 & 56 & -28.48 & $\mathrm{~N}$ & $\mathrm{~N}$ \\
\hline CNH-14+1.75 & 30.5 & 19 & 0.17 & 110 & -27.68 & $\mathrm{~N}$ & 62 \\
\hline $\mathrm{CNH}-14+2.25$ & 31.00 & 28 & 0.15 & 187 & -27.44 & $\mathrm{~N}$ & $\mathrm{~N}$ \\
\hline CNH-14-0.1 & 32.05 & 119 & 0.18 & 658 & -28.15 & -28.98 & 178 \\
\hline $\mathrm{CNH}-15+0.6$ & 32.75 & 12 & 0.22 & 54 & -28.73 & -29.73 & 92 \\
\hline CNH-15+1.6 & 33.75 & 12 & 0.08 & 145 & -27.74 & $\mathrm{~N}$ & 92 \\
\hline CNH-15+2.8 & 34.95 & 26 & 0.24 & 110 & -29.61 & -30.25 & 66 \\
\hline CNH-15-0.3 & 36.55 & $\mathrm{~N}$ & $\mathrm{~N}$ & $\mathrm{~N}$ & -28.61 & $\mathrm{~N}$ & $\mathrm{~N}$ \\
\hline $\mathrm{CNH}-16+0.3$ & 37.15 & 8.7 & 0.13 & 68 & -29.05 & $\mathrm{~N}$ & $\mathrm{~N}$ \\
\hline $\mathrm{CNH}-16+1.3$ & 38.15 & $\mathrm{~N}$ & $\mathrm{~N}$ & $\mathrm{~N}$ & -28.95 & -30.57 & $\mathrm{~N}$ \\
\hline $\mathrm{CNH}-16+2.3$ & 39.15 & 4 & 0.13 & 30 & -30.32 & $\mathrm{~N}$ & $\mathrm{~N}$ \\
\hline CNH-18+0.2 & 46.55 & 2.9 & 0.09 & 32 & -30.84 & $\mathrm{~N}$ & 76 \\
\hline CNH-18+2 & 48.35 & $\mathrm{~N}$ & $\mathrm{~N}$ & $\mathrm{~N}$ & -28.95 & $\mathrm{~N}$ & $\mathrm{~N}$ \\
\hline CNH-18+3.4 & 49.75 & 5.9 & 0.08 & 76 & -27.97 & $\mathrm{~N}$ & 46 \\
\hline
\end{tabular}


Table DR3. Palynofacies analysis data from ZK4703, quantifying the abundance of pollen and spores, plant cuticle, amorphous organic matter and coalified particles.

\begin{tabular}{|c|c|c|c|c|c|c|c|c|c|c|c|c|}
\hline \multirow[b]{2}{*}{ Sample } & \multirow{2}{*}{$\begin{array}{c}\text { Weight } \\
(\mathrm{g})\end{array}$} & \multicolumn{7}{|c|}{ Counts taken across slide transect } & \multirow[b]{2}{*}{$\begin{array}{l}\text { Total phytoclasts } \\
\text { in transect }\end{array}$} & \multirow[b]{2}{*}{$\begin{array}{l}\text { Total phytoclasts } \\
\text { in slide }\end{array}$} & \multirow[b]{2}{*}{$\begin{array}{l}\text { Total phytoclasts } \\
\text { in bulk sample }\end{array}$} & \multirow[b]{2}{*}{ Total phytoclasts /g } \\
\hline & & Wood & Cuticle & Charcoal & $\begin{array}{c}\text { Spore \& } \\
\text { Pollen }\end{array}$ & $\mathrm{AOM}$ & Fungal & $\begin{array}{l}\text { Coalified } \\
\text { material }\end{array}$ & & & & \\
\hline $\mathrm{ZK}-2$ & 46.0 & 23 & 15 & 75 & 5 & 6 & 0 & 301 & 425 & 3400 & 34871795 & 758082 \\
\hline ZK-5 & 39.8 & 36 & 12 & 21 & 29 & 15 & 0 & 133 & 246 & 1968 & 20184615 & 506642 \\
\hline ZK-8 & 39.1 & 8 & 5 & 39 & 4 & 77 & 0 & 211 & 344 & 2752 & 28225641 & 721699 \\
\hline ZK-10 & 38.2 & 59 & 4 & 6 & 13 & 0 & 0 & 144 & 226 & 1808 & 18543590 & 485816 \\
\hline ZK-12 & 39.0 & 29 & 6 & 1 & 8 & 0 & 0 & 69 & 113 & 904 & 30133333 & 772254 \\
\hline ZK-15 & 44.4 & 34 & 3 & 6 & 19 & 0 & 0 & 74 & 136 & 1088 & 36266667 & 816817 \\
\hline ZK-17 & 40.6 & 18 & 3 & 4 & 11 & 1 & 0 & 67 & 104 & 832 & 8320000 & 205078 \\
\hline ZK-19 & 39.5 & 12 & 3 & 1 & 0 & 1 & 0 & 53 & 70 & 560 & 28000000 & 708861 \\
\hline ZK-23 & 39.3 & 9 & 4 & 49 & 2 & 46 & 0 & 141 & 251 & 2008 & 1004000 & 25580 \\
\hline ZK-26 & 41.3 & 1 & 3 & 10 & 0 & 3 & 0 & 40 & 57 & 456 & 22800000 & 551791 \\
\hline ZK-28 & 40.0 & 12 & 4 & 17 & 10 & 3 & 0 & 51 & 97 & 776 & 9700000 & 242682 \\
\hline ZK-30 & 38.6 & 12 & 6 & 18 & 6 & 2 & 0 & 61 & 105 & 420 & 21000000 & 543478 \\
\hline ZK-32 & 39.5 & 5 & 2 & 34 & 3 & 3 & 0 & 76 & 123 & 984 & 49200000 & 1245254 \\
\hline ZK-34 & 39.2 & 45 & 2 & 7 & 20 & 0 & 0 & 101 & 175 & 1400 & 35000000 & 892174 \\
\hline ZK-37 & 39.2 & 44 & 7 & 32 & 18 & 4 & 0 & 241 & 346 & 2768 & 1384000 & 35333 \\
\hline ZK-41 & 39.6 & 16 & 4 & 11 & 76 & 0 & 0 & 623 & 730 & 5840 & 2920000 & 73737 \\
\hline ZK-45 & 40.1 & 3 & 0 & 1 & 3 & 0 & 0 & 310 & 317 & 2536 & 634000 & 15818 \\
\hline
\end{tabular}




\begin{tabular}{|l|l|l|l|l|l|l|l|l|l|l|l|l|}
\hline ZK-49 & 39.2 & 3 & 0 & 3 & 2 & 0 & 0 & 184 & 192 & 1536 & 384000 \\
\hline ZK-53 & 41.3 & 35 & 1 & 11 & 20 & 3 & 0 & 489 & 559 & 4472 & 9803 \\
\hline ZK-63 & 40.6 & 11 & 1 & 65 & 3 & 11 & 0 & 216 & 307 & 1228 & 16675 \\
\hline ZK-65 & 44.4 & 69 & 16 & 156 & 61 & 40 & 0 & 285 & 627 & 5016 & 38000 \\
\hline ZK-69 & 40.7 & 20 & 2 & 10 & 2 & 0 & 0 & 488 & 522 & 4176 & 3721 \\
\hline ZK-73 & 39.4 & 11 & 2 & 162 & 4 & 15 & 0 & 272 & 466 & 3728 & 138 \\
\hline ZK-77 & 39.3 & 43 & 14 & 41 & 9 & 0 & 0 & 64 & 171 & 1368 & 342462 \\
\hline ZK-79 & 39.2 & 10 & 0 & 44 & 8 & 0 & 0 & 43 & 105 & 840 & 84000 \\
\hline ZK-83 & 38.8 & 5 & 0 & 212 & 3 & 0 & 0 & 90 & 310 & 1860 & 87298 \\
\hline
\end{tabular}


Table DR4. Palynofacies analysis data from Chinahe, quantifying the abundance of pollen and spores, plant cuticle, amorphous organic matter and coalified particles.

\begin{tabular}{|c|c|c|c|c|c|c|c|c|c|c|c|c|}
\hline \multirow[b]{2}{*}{ Sample } & \multirow{2}{*}{$\begin{array}{c}\text { Weight } \\
(\mathrm{g})\end{array}$} & \multicolumn{7}{|c|}{ Counts taken across slide transect } & \multirow{2}{*}{$\begin{array}{l}\text { Total } \\
\text { phytoclasts } \\
\text { in transect }\end{array}$} & \multirow{2}{*}{$\begin{array}{l}\text { Total } \\
\text { phytoclasts in } \\
\text { slide }\end{array}$} & \multirow{2}{*}{$\begin{array}{l}\text { Total } \\
\text { phytoclasts in } \\
\text { bulk sample }\end{array}$} & \multirow{2}{*}{$\begin{array}{c}\text { Total } \\
\text { phytoclasts/g }\end{array}$} \\
\hline & & Wood & Cuticle & Charcoal & $\begin{array}{l}\text { Spore \& } \\
\text { Pollen }\end{array}$ & $\mathrm{AOM}$ & Fungal & $\begin{array}{c}\text { Coalified } \\
\text { material }\end{array}$ & & & & \\
\hline $\mathrm{CNH}-3+1$ & 50.1 & 0 & 1 & 32 & 0 & 0 & 0 & 291 & 324 & 1296 & 86400000 & 1724551 \\
\hline $\mathrm{CNH}-4+1.3$ & 50.4 & 0 & 0 & 19 & 0 & 0 & 0 & 276 & 295 & 2360 & 157333333 & 3121693 \\
\hline CNH-6-1 & 51.0 & 3 & 0 & 21 & 0 & 0 & 0 & 137 & 161 & 1288 & 68693333 & 1346928 \\
\hline CNH-9+1.2 & 50.6 & 0 & 0 & 87 & 0 & 0 & 0 & 321 & 408 & 3264 & 130560000 & 2580237 \\
\hline CNH-10+0.4 & 50.1 & 0 & 0 & 2 & 2 & 0 & 0 & 166 & 170 & 1360 & 45333333 & 904857 \\
\hline CNH-12+0.5 & 50.6 & 2 & 0 & 45 & 3 & 0 & 0 & 121 & 171 & 1368 & 85120000 & 1682213 \\
\hline $\mathrm{CNH}-12+1.6$ & 50.5 & 3 & 0 & 132 & 0 & 0 & 0 & 280 & 415 & 3320 & 59022222 & 1168757 \\
\hline CNH-13+0-1 & \multirow{3}{*}{50.6} & 7 & 0 & 86 & 23 & 0 & 0 & 371 & 487 & 3896 & 121208889 & \multirow{3}{*}{2603074} \\
\hline $\mathrm{CNH}-13+0-2$ & & 0 & 0 & 2 & 0 & 0 & 0 & 11 & 13 & 104 & 346667 & \\
\hline CNH-13+0-3 & & 9 & 1 & 31 & 14 & 0 & 0 & 326 & 381 & 3048 & 10160000 & \\
\hline CNH-13+0.3-1 & \multirow{2}{*}{50.4} & 0 & 0 & 4 & 3 & 0 & 0 & 48 & 55 & 55 & 183333 & \multirow{3}{*}{226918} \\
\hline \multirow[t]{2}{*}{$\mathrm{CNH}-13+0.3-2$} & & 7 & 5 & 123 & 47 & 0 & 0 & 240 & 422 & 3376 & 11253333 & \\
\hline & & & & & & & & & & & 11436667 & \\
\hline CNH-13+0.7-1 & \multirow{2}{*}{51.0} & 2 & 2 & 81 & 40 & 0 & 0 & 234 & 359 & 2872 & 102115556 & \multirow{3}{*}{2029560} \\
\hline $\mathrm{CNH}-13+0.7-2$ & & 1 & 3 & 21 & 31 & 0 & 0 & 205 & 261 & 2088 & 1392000 & \\
\hline & & & & & & & & & & & 103507556 & \\
\hline
\end{tabular}




\begin{tabular}{|c|c|c|c|c|c|c|c|c|c|c|c|c|}
\hline CNH-13+1.1 & 50.2 & 1 & 0 & 9 & 16 & 0 & 0 & 97 & 123 & 984 & 69973333 & 1393891 \\
\hline CNH-13+1.5 & 50.4 & 0 & 1 & 8 & 11 & 0 & 0 & 94 & 114 & 912 & 32426667 & 643386 \\
\hline CNH-13+1.9-1 & \multirow{2}{*}{50.4} & 6 & 2 & 56 & 7 & 0 & 0 & 321 & 392 & 3136 & 8362667 & \multirow{3}{*}{479506} \\
\hline \multirow{2}{*}{ CNH-13+1.9-2 } & & 1 & 3 & 10 & 9 & 0 & 0 & 104 & 127 & 1016 & 15804444 & \\
\hline & & & & & & & & & & & 24167111 & \\
\hline CNH-14+0.1 & 50.2 & 0 & 0 & 8 & 2 & 0 & 0 & 76 & 86 & 688 & 15288889 & 304560 \\
\hline CNH-14+1.75 & 50.1 & 0 & 0 & 16 & 0 & 0 & 0 & 232 & 248 & 1984 & 1322667 & 26401 \\
\hline CNH-15+2.6 & 51.2 & 5 & 1 & 14 & 16 & 0 & 0 & 304 & 340 & 2720 & 7253333 & 141667 \\
\hline CNH-16+1.3 & 50.9 & 0 & 1 & 2 & 0 & 0 & 0 & 78 & 81 & 648 & 648000 & 12731 \\
\hline CNH-18+0.2 & 50.3 & 2 & 0 & 10 & 5 & 0 & 0 & 65 & 82 & 656 & 2186667 & 43472 \\
\hline CNH-18+5.3 & 50.9 & 0 & 0 & 11 & 2 & 0 & 0 & 218 & 231 & 1848 & 1848000 & 36306 \\
\hline $\mathrm{CNH}-20+1$ & 50.3 & 0 & 0 & 19 & 2 & 0 & 0 & 101 & 122 & 976 & 15182222 & 301833 \\
\hline $\mathrm{CNH}-21+1.5$ & 50.1 & 0 & 0 & 15 & 0 & 0 & 0 & 120 & 135 & 1080 & 720000 & 14371 \\
\hline
\end{tabular}

